Proyecciones

Vol. 17, $\mathrm{N}^{\circ}$ 2, pp. 227-235, December 1998

Universidad Católica del Norte

Antofagasta - Chile

\title{
ON LAGRANGES POLYNOMIALS OF THREE VARIABLES
}

\author{
MUMTAZ AHMAD KHAN \\ and \\ AJAY KUMAR SHUKLA \\ Aligarh Muslim University, INDIA
}

\begin{abstract}
The present paper introduces a three variables analogue of Lagranges Polynomials and gives certain results involving these polynomials and the four Appell's functions $F_{1}, F_{2}, F_{3}$ and $F_{4}$.
\end{abstract}




\section{Introduction}

Lagrange Polynomials arise in certain problems in statistics. In literature they are denoted by the symbol $g_{n}^{(\alpha, \beta)}(x, y)$ and are defined by means of the following generating relation (see $[2-3]$ ) :

$$
(1-x t)^{-\alpha}(1-y t)^{-\beta}=\sum_{n=0}^{\infty} g_{n}^{(\alpha, \beta)}(x, y) t^{n}
$$

Before we introduce a three variable generalization of $g_{n}^{(\alpha, \beta)}(x, y)$, we first prove the following lemma :

\section{Lemma :}

$$
\sum_{n=0}^{\infty} \sum_{m=0}^{\infty} \sum_{k=0}^{\infty} A(k, m, n)=\sum_{n=0}^{\infty} \sum_{m=0}^{n} \sum_{k=0}^{m} A(k, m-k, n-m)
$$

and

$$
\sum_{n=0}^{\infty} \sum_{m=0}^{n} \sum_{k=0}^{m} A(k, m, n)=\sum_{n=0}^{\infty} \sum_{m=0}^{\infty} \sum_{k=0}^{\infty} A(k, m+k, n+m)
$$

Proof : Consider the series

$$
\sum_{n=0}^{\infty} \sum_{m=0}^{n} \sum_{k=0}^{m} A(k, m, n) t^{n+m+k}
$$

in which $t^{n+m+k}$ has been inserted for convenience and will be removed later by placing $t=1$. Let us collect the powers of $t$ in (1.4). We introduce new indices of summation $j, s$ and $r$ by

$$
k=j, m=s-j, n=r-s
$$

so that the exponenet $(n+m+k)$ in (1.4) becomes $r$. The old indices $n, m$ and $k$ in (1.4) are restricted, as indicated in the summation symbol, by the inequalities

$$
n \geq 0, m \geq 0, k \geq 0
$$

Because of (1.5) the inequalities (1.6) become

$$
r-s \geq 0, s-j \geq 0, j \geq 0
$$


or $0 \leq s \leq r, 0 \leq j \leq s$ with $r$, the exponent on $t$, restricted only in that it must be a non-negative integer. Thus we arrive at

$(1.7) \sum_{n=0}^{\infty} \sum_{m=0}^{n} \sum_{k=0}^{m} A(k, m, n) t^{n+m+k}=\sum_{r=0}^{\infty} \sum_{s=0}^{r} \sum_{j=0}^{s} A(j, s-j, r-s) t^{r}$

and identity (1.2) of the Lemma follows by putting $t=1$ and replacintg the dummy indices $j, s$ and $r$ on the right by dummy indices $k, m$ and $n$.

In the Lemma, equation (1.3) is merely (1.2) written in reverse ; it needs no separate derivation.

Some of the definitions and notations used in this paper are as follows (see $[6])$ :

The binomial coefficient is expressed as

$$
\begin{gathered}
\left(\begin{array}{l}
\lambda \\
n
\end{array}\right)=\frac{\lambda(\lambda-1) \ldots(\lambda-n+1)}{n !}=\frac{(-1)^{n}(-\lambda)_{n}}{n !} \\
(1-z)^{-a}=\sum_{n=0}^{\infty} \frac{{ }^{(a)} n^{z^{n}}}{n !}
\end{gathered}
$$

Appell's four functions of two variables are given by (see [4])

$$
F_{1}\left(a ; b, b^{\prime} ; c ; x, y\right)=\sum_{n, k=0}^{\infty} \frac{(a)_{n+k}(b)_{n}\left(b^{\prime}\right)_{k} x^{n} y^{k}}{n ! k !(c)_{n+k}}
$$

$$
\begin{gathered}
F_{2}\left(a ; b, b^{\prime} ; c, c^{\prime} ; x, y\right)=\sum_{n, k=0}^{\infty} \frac{(a)_{n+k}(b)_{n}\left(b^{\prime}\right)_{k} x^{n} y^{k}}{n ! k !(c)_{n}\left(c^{\prime}\right)_{k}} \\
F_{3}\left(a, a^{\prime} ; b, b^{\prime} ; c ; x, y\right)=\sum_{n, k=0}^{\infty} \frac{(a)_{n}\left(a^{\prime}\right)_{k}(b)_{n}\left(b^{\prime}\right)_{k} x^{n} y^{k}}{n ! k !(c)_{n+k}} \\
F_{4}\left(a, b ; c, c^{\prime} ; c ; x, y\right)=\sum_{n, k=0}^{\infty} \frac{(a)_{n+k}(b)_{n+k} x^{n} y^{k}}{n ! k !\left(c^{\prime}\right)_{k}}
\end{gathered}
$$

For definitions of various cuadruple hypergeometric functions see $\mathrm{H}$. Exton [1]. However, the Lauricella function $F_{D}^{(4)}$ of four variables is defined as (see [5])

$$
F_{D}^{(4)} a, b_{1}, b_{2}, b_{3}, b_{4} ; c ; x_{1}, x_{2}, x_{3}, x_{4}
$$




$$
=\sum_{k, m, n, p=0}^{\infty} \frac{(a)_{k+m+n+p}^{\left(b_{1}\right)_{k}\left(b_{2}\right)_{m}\left(b_{3}\right)_{n}\left(b_{4}\right)_{p}}}{(c)_{k+m+n+p}} \frac{x_{1}^{k}}{k !} \frac{x_{2}^{m}}{m !} \frac{x_{3}^{n}}{n !} \frac{x_{4}^{p}}{p !}
$$

The quadruple hypergeometric function $K_{11}$ and $K_{15}$ are defined as (see [1])

$K_{11}\left(a, a, a, a ; b_{1}, b_{2}, b_{3}, b_{4} ; c, c, c, d ; x, y, z, t\right)$

$$
\begin{aligned}
& (1.15)=\sum_{k, m, n, p=0}^{\infty} \frac{(a)_{k+m+n+p}\left(b_{1}\right)_{k}\left(b_{2}\right)_{m}\left(b_{3}\right)_{n}\left(b_{4}\right)_{p}}{k ! m ! n ! p !(c)_{k+m+n}(d)_{p}} x^{k} y^{m} z^{n} t^{p} \\
& K_{15}\left(a, a, a, b_{5} ; b_{1}, b_{2}, b_{3}, b_{4} ; c, c, c, c ; x, y, z, t\right) \\
& (1.16) \sum_{k, m, n, p=0}^{\infty} \frac{(a)_{k+m+n+p}\left(b_{1}\right)_{k}\left(b_{2}\right)_{m}\left(b_{3}\right)_{n}\left(b_{4}\right)_{p}\left(b_{5}\right)_{p}}{k ! m ! n ! p !(c)_{k+m+n+p}} x^{k} y^{m} z^{n} t^{p}
\end{aligned}
$$

\section{Lagrange Polynomials of Three Variables}

We define the three variable analogue of Lagrange Polynomials $g_{n}^{(\alpha, \beta, \gamma)}(x, y, z)$ by means of the following generating relation :

$$
(1-x t)^{-\alpha}(1-y t)^{-\beta}(1-z t)^{-\gamma}=\sum_{n=0}^{\infty} g_{n}^{(\alpha, \beta, \gamma)}(x, y, z) t^{n}
$$

Expanding the L. H. S. of (2.1) by (1.9), using the result of the lemma proved and finally equating the coefficient of $t^{n}$, one gets

$$
g_{n}^{(\alpha, \beta, \gamma)}(x, y, z)=\sum_{k=0}^{n} \sum_{p=0}^{k} \frac{(\alpha)_{n-k}(\beta)_{k-p}(\gamma)_{p}}{(n-k) !(k-p) ! p !} x^{n-k} y^{k-p} z^{p}
$$

Polynomials $g_{n}^{(\alpha, \beta, \gamma)}(x, y, z)$ can be regarded as a generalization of Lagrange Polynomials from two to three variables as it can easily be seen that

$$
g_{n}^{(\alpha, \beta, \gamma)}(x, y, 0)=g_{n}^{(\alpha, \beta)}(x, y)
$$

Also,

$$
\begin{gathered}
g_{n}^{(\alpha, \beta, \gamma)}(x, y, y)=g_{n}^{(\alpha, \beta+\gamma)}(x, y) \\
g_{n}^{(\alpha, \beta, \gamma)}(x, y, z)=g_{n}^{(\alpha+\gamma, \beta)}(x, y) \\
g_{n}^{(\alpha, \beta, \gamma)}(x, y, z)=g_{n}^{(\alpha+\beta, \gamma)}(x, y) \\
g_{n}^{(\alpha, \beta, 0)}(x, y, z)=g_{n}^{(\alpha, \beta)}(x, y)
\end{gathered}
$$


Now consider

$$
\begin{gathered}
\sum_{n=0}^{\infty} g_{n}^{(a+\alpha+\lambda, b+\beta+\mu, c+\gamma+\nu)}(x, y, z) t^{n} \\
=(1-x t)^{-(a+\alpha+\lambda)}(1-y t)^{-(b+\beta+\mu)}(1-z t)^{-(c+\gamma+\nu)} \\
=\sum_{n=0}^{\infty} \sum_{m=0}^{\infty} \sum_{k=0}^{\infty} g_{n}^{(a, b, c)}(x, y, z) g_{m}^{(\alpha, \beta, \gamma)}(x, y, z) g_{k}^{(\lambda, \mu, \nu)}(x, y, z) t^{n+m+k} \\
=\sum_{n=0}^{\infty} \sum_{m=0}^{n} \sum_{k=0}^{m} g_{n-m}^{(a, b, c)}(x, y, z) g_{m-k}^{(\alpha, \beta, \gamma)}(x, y, z) g_{k}^{(\lambda, \mu, \nu)}(x, y, z) t^{n}
\end{gathered}
$$

using (1.2).

Now equating the coefficient of $t^{n}$, we get $g_{n}^{(a+\alpha+\lambda, b+\beta+\mu, c+\gamma+\nu)}(x, y, z)$

$$
=\sum_{m=0}^{n} \sum_{k=0}^{m} g_{n-m}^{(a, b, c)}(x, y, z) g_{m-k}^{(\alpha, \beta, \gamma)}(x, y, z) g_{k}^{(\lambda, \mu, \nu)}(x, y, z)
$$

As a particular case of (2.8) it can easily be verified that

$$
g_{n}^{\left(\alpha+\alpha^{\prime}, \beta+\beta^{\prime}, \gamma+\gamma^{\prime}\right)}(x, y, z)
$$

$$
\sum_{r=0}^{n} g_{n-r}^{(\alpha, \beta, \gamma)}(x, y, z) g_{r}^{\left(\alpha^{\prime}, \beta^{\prime}, \gamma^{\prime}\right)}(x, y, z)
$$

\section{Main Results}

First consider the series

$$
\begin{aligned}
& \sum_{n=0}^{\infty} \sum_{m=0}^{\infty} \sum_{k=0}^{\infty} \frac{(n+m+k) !}{n ! m ! k !} g_{n+m+k}^{(\alpha, \beta, \gamma)}(x, y, z) t^{n} v^{m} w^{k} \\
& =\sum_{n=0}^{\infty} \sum_{m=0}^{n} \sum_{k=0}^{m} \frac{(-n)_{m}(-m)_{k}}{m ! k !} g_{n}^{(\alpha, \beta, \gamma)}(x, y, z) t^{n}\left(-\frac{v}{t}\right)^{m}\left(-\frac{w}{v}\right)^{k}
\end{aligned}
$$

by $(1.2)$

$$
\begin{aligned}
& =\sum_{n=0}^{\infty} \sum_{m=0}^{n} \frac{(-n)_{m}}{m !} g_{n}^{(\alpha, \beta, \gamma)}(x, y, z) t^{n}\left(-\frac{v}{t}\right)^{m}\left(1+\frac{w}{v}\right)^{m} \text { by } \\
& =\sum_{n=0}^{\infty} g_{n}^{(\alpha, \beta, \gamma)}(x, y, z)(t+v+w)^{n} \\
& =(1-x t-x v)^{-\alpha}(1-y t-y v)^{-\beta}(1-z t-z v)^{-\gamma} \\
& \sum_{k=0}^{\infty} g_{k}^{(\alpha, \beta, \gamma)}\left(\frac{x}{1-x t-x v}, \frac{y}{1-y t-y v}, \frac{z}{1-z t-z v}\right) w^{k}
\end{aligned}
$$


Equating the coefficient of $w^{k}$, we arrive at

$$
\begin{aligned}
& \sum_{n=0}^{\infty} \sum_{m=0}^{\infty} \frac{(n+m+k) !}{n ! m ! k !} g_{n+m+k}^{(\alpha, \beta, \gamma)}(x, y, z) t^{n} v^{m} \\
& =(1-x t-x v)^{-\alpha}(1-y t-y v)^{-\beta}(1-z t-z v)^{-\gamma}
\end{aligned}
$$

$$
g_{k}^{(\alpha, \beta, \gamma)}\left(\frac{x}{1-x t-x v}, \frac{y}{1-y t-y v}, \frac{z}{1-z t-z v}\right)
$$

As a simpler version of (3.1), we have

$$
\begin{aligned}
& \sum_{n=0}^{\infty}\left(\begin{array}{c}
m+n \\
n
\end{array}\right) g_{m+n}^{(\alpha, \beta, \gamma)}(x, y, z) t^{n} \\
= & (1-x t)^{-\alpha}(1-y t)^{-\beta}(1-z t)^{-\gamma} g_{m}^{(\alpha, \beta, \gamma)}\left(\frac{x}{1-x t}, \frac{y}{1-y t}, \frac{z}{1-z t}\right)
\end{aligned}
$$

Next, consider

$$
\begin{aligned}
& \sum_{n=0}^{\infty} g_{n}^{(\alpha, \beta, \gamma)}(x, y, z) F_{2}[\lambda ;-n, \delta ; \mu, \nu ; u, u w] t^{n} \\
& \sum_{n=0}^{\infty} \sum_{q=0}^{\infty} \sum_{p=0}^{\infty} \frac{(\lambda)_{p+q}(n+p) !(\delta)_{q}}{n ! p ! q !(\mu)_{p}(\nu)_{q}} g_{n+p}^{(\alpha, \beta, \gamma)}(x, y, z)(-u)^{p}(-u w)^{q} t^{n+p} \\
& \sum_{q=0}^{\infty} \sum_{p=0}^{\infty} \frac{(\lambda)_{p+q}(\delta)_{q}}{q !(\mu)_{p}(\nu)_{q}}(-u t)^{p}(u w)^{q}(1-x t)^{-\alpha}(1-v t)^{-\beta}(1-z t)^{-\gamma} \\
& g_{n+p}^{(\alpha, \beta, \gamma)}\left(\frac{x}{1-x t}, \frac{y}{1-y t}, \frac{z}{1-z t}\right) \text { by }(3.2) \\
& =(1-x t)^{-\alpha}(1-y t)^{-\beta}(1-z t)^{-\gamma} \sum_{q=0}^{\infty} \sum_{p=0}^{\infty} \sum_{k=0}^{\infty} \sum_{j=0}^{\infty} \frac{(\lambda)_{p+q+k+j}}{(\mu)_{p+k+j}(\nu)_{q}} \\
& \frac{(\alpha)_{p}(\beta)_{k}(\gamma)_{j}(\delta)_{q}\left(\frac{x u t}{x t-1}\right)^{p}\left(\frac{y u t}{y t-1}\right)^{k}\left(\frac{z u t}{z t-1}\right)^{j}(u w)^{q}}{p ! q ! j !}
\end{aligned}
$$

Thus we arrive at the result

$$
\begin{aligned}
& \sum_{n=0}^{\infty} g_{n}^{(\alpha, \beta, \gamma)}(x, y, z) F_{2}[\lambda ;-n, \delta ; \mu, \nu ; u, u w] t^{n} \\
& =(1-x t)^{-\alpha}(1-y t)^{-\beta}(1-z t)^{-\gamma}
\end{aligned}
$$

(3.3) $K_{11}\left(\lambda, \lambda, \lambda, \lambda ; \alpha, \beta, \gamma, \delta ; \mu, \mu, \mu, \nu ; \frac{x u t}{x t-1}, \frac{y u t}{y t-1}, \frac{z u t}{z t-1}, u w\right)$ 
We then consider

$$
\begin{aligned}
& \sum_{n=0}^{\infty} g_{n}^{(\alpha, \beta, \gamma)}(x, y, z) F_{1}[\lambda ;-n, \delta ; \mu, u, u w] t^{n} \\
& =\sum_{q=0}^{\infty} \sum_{p=0}^{\infty} \frac{(\lambda)_{p+q}(\delta)_{q}}{q !(\mu)_{p+q}}(-u t)^{p}(u w)^{q} \sum_{n=0}^{\infty}\left(\begin{array}{c}
n+p \\
n
\end{array}\right) g_{n+p}^{(\alpha, \beta, \gamma)}(x, y, z) t^{n} \\
& =(1-x t)^{-\alpha}(1-y t)^{-\beta}(1-z t)^{-\gamma} \sum_{p=0}^{\infty} \sum_{q=0}^{\infty} \sum_{k=0}^{\infty} \sum_{j=0}^{\infty} \frac{(\lambda)_{p+q+k+j}}{(\mu)_{p+q+k+j}} \\
& \frac{(\alpha)_{p}(\beta)_{k}(\gamma)_{j}(\delta)_{q}}{p ! q ! k ! j ! q !}\left(\frac{x u t}{x t-1}\right)^{p}\left(\frac{y u t}{y t-1}\right)^{k}\left(\frac{z u t}{z t-1}\right)^{j}(u w)^{q}
\end{aligned}
$$

Thus, we have

$$
\sum_{n=0}^{\infty} g_{n}^{(\alpha, \beta, \gamma)}(x, y, z) F_{1}[\lambda ;-n, \delta ; \mu, u, u w] t^{n}
$$$$
=(1-x t)^{-\alpha}(1-y t)^{-\beta}(1-z t)^{-\gamma} F_{D}^{(4)}\left(\lambda, \alpha, \beta, \gamma, \delta ; \mu \frac{x u t}{x t-1}, \frac{y u t}{y t-1}, \frac{z u t}{z t-1}, u w\right)
$$

$$
\text { Next, we consider }
$$

$$
\begin{aligned}
& \sum_{n=0}^{\infty} g_{n}^{(\alpha, \beta, \gamma)}(x, y, z) F_{3}[\lambda,-n ; \delta, \mu ; \rho ; u, u w] t^{n} \\
= & \sum_{p=0}^{\infty} \sum_{q=0}^{\infty} \frac{(\lambda)_{q}(\delta)_{q}(\mu)_{p}}{q !(\rho)_{p+q}}(-u t)^{p}(u w)^{q} \sum_{n=0}^{\infty}\left(\begin{array}{c}
n+p \\
n
\end{array}\right) g_{n+p}^{(\alpha, \beta, \gamma)}(x, y, z) t^{n} \\
= & (1-x t)^{-\alpha}(1-y t)^{-\beta}(1-z t)^{-\gamma} \sum_{p=0}^{\infty} \sum_{q=0}^{\infty} \frac{(\lambda)_{q}(\delta)_{q}(\mu)_{p}}{q !(\rho)_{p+q}}(-u t)^{p}(u w)^{q} \\
& \sum_{k=0}^{p} \sum_{j=0}^{k} \frac{(\alpha)_{p-k}(\beta)_{k-j}(\gamma)_{j}}{(p-k) !(k-j) ! j !}\left(\frac{x}{1-x t}\right)^{p-k}\left(\frac{y}{1-y t}\right)^{k-j}\left(\frac{z}{1-z t}\right)^{j} \text { by }
\end{aligned}
$$

Thus, we obtain

$$
\begin{gathered}
\sum_{n=0}^{\infty} g_{n}^{(\alpha, \beta, \gamma)}(x, y, z) F_{3}[\lambda,-n ; \delta, \mu ; \rho ; u, u w] t^{n} \\
=(1-x t)^{-\alpha}(1-y t)^{-\beta}(1-z t)^{-\gamma} \\
\text { (3.5) } K_{15}\left(\mu, \mu, \mu, \lambda ; \alpha, \beta, \gamma, \delta ; \rho, \rho, \rho, \rho ; \frac{x u t}{x t-1}, \frac{y u t}{y t-1}, \frac{z u t^{j}}{z t-1}, u w\right)
\end{gathered}
$$


Finally, we consider

$$
\begin{aligned}
& \sum_{n=0}^{\infty} \frac{(\lambda)_{n}(\delta)_{n}}{(1-\alpha)_{n}(1-\beta)_{n}(1-\gamma)_{n}} g_{n}^{(\alpha-n, \beta-n, \gamma-n)}(x, y, z) \\
& F_{4}[\lambda+n ; \delta+n ; \mu, \rho ; u, w] t^{n} \\
& =\sum_{n=0}^{\infty} \sum_{p=0}^{\infty} \sum_{q=0}^{\infty} \frac{(\lambda)_{n+p+q}(\delta)_{n+p+q}}{p ! q !(\mu)_{p}(\rho)_{q}} u^{p} w^{q} \sum_{k=0}^{n} \sum_{j=0}^{k} \frac{(\alpha)_{-k}(\beta)_{-n+k-j}}{(n-k) !} \\
& \frac{(\gamma)_{-n+j}}{(k-j) ! j !}(-1)^{n} x^{n-k} y^{k-j} z^{j} t^{n} \\
& =\sum_{n=0}^{\infty} \sum_{p=0}^{\infty} \sum_{q=0}^{\infty} \sum_{k=0}^{p} \sum_{j=0}^{k} \\
& \frac{(\lambda)_{n+k+j+p+q}(\delta)_{n+k+j+p+q}(-x t)^{n}(-y t)^{k}(-z t)^{j} u^{p} w^{q}}{p ! q ! n ! k ! j !(\mu)_{p}(\rho)_{q}(1-\alpha)_{k+j}(1-\beta)_{n+j}(1-\gamma)_{n+k}}
\end{aligned}
$$$$
\sum_{n=0}^{\infty} \frac{(\lambda)_{n}(\delta)_{n}}{(1-\alpha)_{n}(1-\beta)_{n}(1-\gamma)_{n}} g_{n}^{(\alpha-n, \beta-n, \gamma-n)}(x, y, z)
$$$$
F_{4}[\lambda+n ; \delta+n ; \mu, \rho, u, w] t^{n}
$$

$$
=F\left(\begin{array}{c}
\lambda, \lambda, \lambda, \lambda, \lambda, \delta, \delta, \delta, \delta, \delta ; \mu, \rho, 1-\alpha, 1-\alpha, 1-\beta, 1-\beta, \\
1-\gamma, 1-\gamma ;-x t,-y t,-z t, u, w
\end{array}\right)
$$

where $F$ is a quintuple hypergeometric function.

\section{References}

[1] H. EXTON, Multiple Hupergeometric Functions and Applications. John Wiley and Sons (Halsted Press), New York ; Ellis Horwood, Chichester (1976).

[2] K. C. MITTAL, Some New Generating Functions for Lagrange's Polynomials Mathematica (Cluj), 44, pp. 57-62, (1979).

[3] K. C. MITTAL and B. L. SHARMA, Some Formulae for Lagranges Polynomials. I and II. Mathematica (Cluj), 9 (32), pp. 311-319, (1967). 
[4] E. D. RAINVILLE, Special Functions. MacMillan, New York; Reprinted by Chelsea Publ. Co., Bronx, New York, (1971).

[5] H. M. SRIVASTAVA and P. W. KARLSSON, Multiple Gausian Hypergeometric Series. John Wiley and Sons (Halsted Press), New York ; Ellis Horwood Chichester, (1985).

[6] H. M. SRIVASTAVA and H. L. MANOCHA, A Treatise on Generating Functions. John Wiley and Sons (Halsted Press), New York ; Ellis Horwood Chichester, (1984).

Received : July 1997.

\section{Mumtaz Ahmad Khan}

and
Ajay Kumar Shukla
Department of Applied Mathematics
Faculty of Engineering
Aligarh Muslim University
Aligarh - 202002, U. P.
INDIA 